\title{
Influence of the interaction between parental myopia and poor reading and writing habits on prevalence of myopia in school students in Urumqi, China
}

\author{
Haonan Shi \\ School of Public Health, Xinjiang Medical University \\ Jing Fu \\ Department of Ophthalmology, First Affiliated Hospital; Xinjiang Medical University \\ Xiaojing Liu \\ Health Care Guidance Centre in Primary and Secondary Schools \\ Yingxia Wang \\ School of Nursing \& Health Management, Shanghai University of Medicine \& Health Sciences \\ Xianting Yong \\ School of Public Health, Xinjiang Medical University \\ Lan Jiang \\ Maternal and Child Health Care Hospital of Uygur Autonomous Region \\ Shaowei Ma \\ School of Public Health, Xinjiang Medical Unversity \\ Zhe Yin \\ School of Public Health, Xinjiang Medical University \\ Jian Yao \\ School of Public Health, Xinjiang Medical University \\ Xuan Yao \\ College of Medicine, Shanghai University \\ Xueyi Chen ( $\sim$ 2339704397@qq.com ) \\ Department of Ophthalmology;First Affiliated Hospital;Xinjiang Medical Unoversity \\ Tingting Wang \\ School of Nursing \& Health Management, Shanghai University of Medicine \& Health Sciences
}

\section{Research article}

Keywords: myopia, heritability, poor reading and writing habits, interaction

Posted Date: March 18th, 2021

DOI: https://doi.org/10.21203/rs.3.rs-330190/v1

License: (c) (i) This work is licensed under a Creative Commons Attribution 4.0 International License. Read Full License

Version of Record: A version of this preprint was published at BMC Ophthalmology on August 14th, 2021. See the published version at https://doi.org/10.1186/s12886-021-02058-3. 


\section{Abstract}

Background: To evaluate the prevalence of myopia in school students in Urumqi, China, and explore the influence of the interaction between parental myopia and poor reading and writing habits on myopia to identify the at-risk population and provide evidence to help school students avoid developing myopia.

Methods: A cross-sectional survey was conducted with 6,883 school students aged 7-20 years in Urumqi in December 2019. The Standard Eye Chart and mydriatic optometry were used to determine whether students had myopia. Falconer's method was used to calculate the heritability of parental myopia. Multivariate unconditional logistic regression models were used to analyze the risk factors for myopia and the additive and multiplicative interaction of parental myopia and poor reading and writing habits.

Results After standardizing the age of the 6,883 students, the overall prevalence rate of myopia was $47.50 \%$. The heritability of parental myopia was $66.57 \%$ for boys, $67.82 \%$ for girls, $65.02 \%$ for the Han group, and $52.71 \%$ for other ethnicities. There were additive interactions between parental myopia and poor reading and writing habits; among them, parental myopia and poor reading and writing habits (1) (the distance between the eyes and book is less than $30 \mathrm{~cm}$ when reading and writing, fingers block the sight of one eye while holding the pen, and leaning one's body when reading and writing) increased the risk of myopia by 10.99 times (odds ratio $[O R]=10.99,95 \%$ confidence interval $[C l]=8.33-14.68$ ), parental myopia and poor reading and writing habits (2) (reading while lying down, walking, or in the car) increased the risk of myopia by 5.92 times $(O R=5.92,95 \% \mathrm{Cl}=4.84-7.27)$. There was no multiplicative interaction between parental myopia and poor reading and writing habits (1) or (2) $(O R=0.69,95 \% C l=0.44-1.08 ; O R=0.89,95 \% C l=0.66-1.21$, respectively).

Conclusion The prevalence of myopia among students in Urumqi, Xinjiang is relatively high. The risk of developing myopia is affected by parental myopia and poor reading and writing habits. In addition, parental myopia amplifies the harm caused by poor reading and writing habits, thereby increasing the risk of myopia. Students with parents who have myopia should be targeted during myopia prevention efforts.

\section{Background}

Myopia is a type of refractive error characterized by worsened distance vision caused by elongation of the axis oculi [1] With the increase in study-induced stress and the popularity of mobile electronic equipment, the prevalence of myopia has increased among school students; this phenomenon is particularly obvious in Southeast Asian countries such as China, Japan, Singapore, and Korea [2]. A survey on health and nutrition from Korea reported that $65.4 \%$ of the population aged 5-18 years in Korea had myopia, and 8.0\% of them had severe myopia in 2016-2017 [3]. A survey of 1416 school students from Japan showed that the prevalence rates of myopia in elementary school and junior high school students were $76.5 \%$ and $94.9 \%$, respectively [4]. The prevalence of myopia in the Chinese was also high; a survey of the prevalence of myopia among school students in six cities showed that the overall prevalence of myopia was $55.7 \%$ [5]. Although the prevalence of myopia is lower in the Chinese than in Korean and Japanese populations, it is still not possible to ignore the detrimental effects of myopia in the Chinese, especially in school students. In addition, we checked the results of the physique and health survey of Chinese students from 2000 to 2014; it is evident that the prevalence of poor vision is increasing in Chinese students, with myopia as the most common issue $[6,7,8,9]$, which gradually affects younger students [10]. Therefore, the new cases of myopia demonstrate that prevention of myopia has worsened.

Genetic and environmental factors are major factors in myopia and have been accepted by most scholars [11, 12]. Recently, many studies have verified these views $[13,14]$. A survey of 7,681 primary and secondary school students in Yunnan, China, a province with a multi-ethnic population, showed that the prevalence of myopia in Dai groups was higher than that in other ethnic groups (Han, Bai, Yi, and other ethnicities) [15]. Similar to Yunnan, Xinjiang is a region where 
multiple ethnic groups, e.g., Han, Uygur, Kazakh, and Hui, live with great differences in their lifestyles, which has caused large differences in the prevalence of myopia in different ethnic groups. However, the results opposed those obtained in Yunnan; the difference in Xinjiang was mainly manifested between the Han population and other ethnic groups. Our research team previously carried out a survey of myopia in school students in 2012 and 2016 . The survey involved two cities in Xinjiang, Urumqi and Yining. The results of this research show that the prevalence of myopia in the Han population was higher than that in other ethnic groups $[16,17,18]$. To understand the change in the myopia rate in students in Urumqi and Xinjiang and the influence of parents' myopia and poor reading and writing habits, we conducted a survey again in December 2019. Through this survey, we hoped to be able to explore the population susceptible to myopia and advise schools and parents to prevent students' myopia.

\section{Methods}

\section{Study population}

This study was a school-based eye survey conducted in Urumqi, the capital city of Xinjiang, in December 2019. There are seven districts and one county in Urumqi. According to the statistics at the end of 2018, Urumqi had a population of approximately 3.5 million, which included Han, Uighur, Kazakh, Hui, and other ethnicities.

At first, we contacted the Primary and Secondary School Health Care Guidance Center and Education Bureau in Urumqi, obtained their approval, and asked school doctors in each school for their assistance. Next, we used stratified cluster sampling to randomly select four districts (Xinshi, Tianshan, Shayibaket, and Shuimogou) in Urumqi. We randomly selected students from the second grade of primary school to the second grade of high school from two to three schools in each district; a total of 7,587 students were initially included in our study. After excluding those who did not meet the inclusion criteria and who had incomplete data, 6,883 (90.72\%) school students participated in our study.

Our research adhered to the tenets of the Declaration of Helsinki for research involving human subjects and was approved by the Institutional Review Board of the First Affiliated Hospital of Xinjiang Medical University. The research methods were carried out in accordance with the approved guidelines. Written informed consent was obtained from all participating students, parents, and the head teacher, and we promised to keep their information confidential.

\section{Clinical eye examination}

The ophthalmic examinations were conducted in each school by school doctors and an Optometrist from the First Affiliated Hospital of Xinjiang Medical University. First, school doctors used a new national standard visual acuity chart to examine uncorrected eyesight in students. Students who had uncorrected eyesight $\geq 5.0$ according to the International Standard Eyesight Chart were classed as emmetropic; students with uncorrected eyesight < 5.0 underwent optometry (streak retinoscope YZ24 [Suzhou Liuliu Vision Technology Co., Ltd.\{http://www.66vision.com\}]) after mydriatic eye drops were administered (tropicamide, purchased from Tianjin Jinyao Group Hebei Yongguang Pharmaceutical Co. Ltd. [http://www.ieye.com.cn]). Students with SE <-0.50 diopters were diagnosed with myopia [19]. If there was a difference between the two eyes, the eye with poorer vision was chosen as the standard. Students with hyperopia, amblyopia, ocular trauma, trachoma, anisometropia, corneal disease, glaucoma, cataract, uveitis, retinal diseases, or other ocular diseases were excluded. In addition, students with diabetes, hyperthyroidism, and endocrine-related metabolic diseases were also excluded.

\section{Measurement of covariates}

We designed a questionnaire to examine the characteristics of students in Xinjiang with the help of head teachers and school students. All students who agreed to participate in the survey answered the questionnaire in a classroom at a class meeting. The questionnaire included the following: (1) general demographic characteristics (sex, ethnicity, age, grade, 
family history of myopia, parental education, family income, place of residence [urban or rural]); (2) vision-related conditions (age when first developed myopia, frequency of changing glasses, history of previous eye diseases, etc.); and (3) eye use (reading and writing habits; frequency of using electronic products such as computers, smart phones, tablets; sleeping time; outdoor activity time; etc.).

\section{Judgment criteria for other indicators}

Poor reading and writing habits (1) was defined as maintaining a distance of $<30 \mathrm{~cm}$ between the eyes and book while reading and/or writing, covering the sight of one eye with the finger holding a pen while writing, and/or tilting the body when reading and/or writing.

Poor reading and writing habits (2) was defined as reading while lying down, walking, or in a moving car.

\section{Statistical analysis}

The data were input using Epi Data 3.1 software. The chi-square test was used to examine differences in the prevalence of myopia stratified by sex, ethnicity, age, parental myopia, and poor reading and writing habits; multivariate logistic regression was used to analyze the associations of parental myopia and poor reading and writing habits with myopia, controlling for age, sex, ethnicity, and other confounding factors. The multiplicative and additive interactions between them were also analyzed. In all statistical analyses, two-tailed tests and a 5\% significance level were applied. The analyses were performed using R Studio.

Parental heritability was examined using Falconer's formula $\left(h^{2}=b / r\right)$; in this formula, $r$ is the correlation coefficient (parents are first-degree relatives, $r=0.5$ ), $b$ is the regression coefficient, $b=p_{\mathrm{c}}\left(X_{\mathrm{c}}-X_{\mathrm{r}}\right) / a_{\mathrm{r}}, p_{\mathrm{c}}=1-q_{\mathrm{c}}$, where $q_{\mathrm{c}}$ is the prevalence of myopia in relatives in the control group, $X_{\mathrm{c}}$ is the standard deviation between the average susceptibility of relatives in the control group and the threshold, $X_{r}$, is the standard deviation between the average susceptibility of relatives in the proband group and the threshold, $a_{r}$, is the standard deviation between the average susceptibility of patients and their relatives in the proband. The values of $X_{\mathrm{c}}, X_{\mathrm{r}}$, and $a_{\mathrm{r}}$ can be found in the Falconer table.

\section{Results}

A total of 6,883 students comprising 3,404 (50.5\%) boys and 3,479 (49.5\%) girls aged 7-20 years (mean \pm standard deviation: $12.42 \pm 2.46$ years) completed the ophthalmic examinations and were subsequently included in this analysis. The study was composed of a multi-ethnic population including Han $(3,564,51.8 \%)$ and other ethnicities $(3,319,48.2 \%)$. Considering all study participants after age standardization, $47.50 \%$ were affected by myopia. The prevalence of myopia was higher in girls than in boys (43.5\% vs. $33.6 \%)$ and higher in the Han population than in the other ethnic groups (53.0\% vs. $23.2 \%)$. Myopia prevalence increased significantly with increasing age and learning level $(P$ for trend $<0.01)$. The prevalence was the lowest in students aged $7-12$ years (19.9\%) and in primary school students (19.9\%) and the highest among those aged $15-20$ years (71.0\%) and in senior high school students (72.0\%). More students with myopia had poor reading and writing habits (1) and (2) (42.3\% and $42.8 \%$, respectively). The prevalence of myopia in students was higher in those with both parents with myopia than in those with only one parent with myopia and those with no parents with myopia (74.3\% vs. $55.2 \%$ vs. $29.6 \%$, respectively) (Table 1 ). 
Table 1

Prevalence of myopia in students by sex, ethnicity, age, learning level, parental myopia, and poor reading and writing habits

\begin{tabular}{|c|c|c|c|c|c|}
\hline Parameters & Number & Myopia & Prevalence(\%) & $x^{2}$ & $P$ \\
\hline \multicolumn{6}{|l|}{ Sex } \\
\hline Male & 3404 & 1145 & 33.6 & \multirow[t]{2}{*}{70.86} & \multirow[t]{2}{*}{$<0.01$} \\
\hline Female & 3479 & 1514 & 43.5 & & \\
\hline \multicolumn{6}{|l|}{ Ethnicity } \\
\hline Han & 3564 & 1889 & 53.0 & \multirow[t]{2}{*}{643.85} & \multirow[t]{2}{*}{$<0.01$} \\
\hline Other nationalities & 3319 & 770 & 23.2 & & \\
\hline \multicolumn{6}{|l|}{ Age (years) } \\
\hline $7-11$ & 3508 & 698 & 19.9 & \multirow[t]{3}{*}{1179.06} & \multirow[t]{3}{*}{$<0.01$} \\
\hline $12-14$ & 2243 & 1157 & 51.6 & & \\
\hline $15-20$ & 1132 & 804 & 71.0 & & \\
\hline \multicolumn{6}{|l|}{ Learning level } \\
\hline Primary school & 3093 & 530 & 19.9 & \multirow[t]{3}{*}{1307.97} & \multirow[t]{3}{*}{$<0.01$} \\
\hline Junior high school & 2475 & 1182 & 44.5 & & \\
\hline Senior high school & 1315 & 947 & 72.0 & & \\
\hline \multicolumn{6}{|c|}{ Poor reading and writing habits (1) } \\
\hline Yes & 5965 & 2522 & 42.3 & \multirow[t]{2}{*}{251.13} & \multirow[t]{2}{*}{$<0.01$} \\
\hline No & 918 & 137 & 14.9 & & \\
\hline \multicolumn{6}{|c|}{ Poor reading and writing habits (2) } \\
\hline Yes & 5181 & 2271 & 42.8 & \multirow[t]{2}{*}{239.14} & \multirow[t]{2}{*}{$<0.01$} \\
\hline No & 1702 & 388 & 22.8 & & \\
\hline \multicolumn{6}{|l|}{ Parental myopia } \\
\hline Neither & 4739 & 1402 & 29.6 & \multirow[t]{3}{*}{573.69} & \multirow[t]{3}{*}{$<0.01$} \\
\hline Either & 1762 & 973 & 55.2 & & \\
\hline Both & 382 & 284 & 74.3 & & \\
\hline
\end{tabular}

Among the 13,766 parents, $18.3 \%$ had myopia. The heritability of parental myopia for boys was $66.57 \%$ and that for girls was $67.82 \%$; the heritability of parental myopia for the Han group was $65.02 \%$ and that for other ethnic groups was $52.71 \%$ (Table 2). 
Table 2

Analysis of heritability of myopia using Falconer's method

\begin{tabular}{|c|c|c|c|c|c|c|c|c|c|c|}
\hline & & $\begin{array}{l}\text { Number } \\
\text { of } \\
\text { parents }\end{array}$ & $\begin{array}{l}\text { Number } \\
\text { with } \\
\text { myopia }\end{array}$ & $\begin{array}{l}\text { Prevalence } \\
\text { of } \\
\text { myopia(\%) }\end{array}$ & $x^{2}$ & $P$ & $q$ & $x$ & $a$ & $\begin{array}{l}h^{2} \\
(\%)\end{array}$ \\
\hline \multicolumn{6}{|l|}{ Sex } & \multirow{5}{*}{$\begin{array}{l}<.01 \\
0.01\end{array}$} & & & & \\
\hline \multirow[t]{2}{*}{ Boys } & Emmetropia ${ }^{1)}$ & 4518 & 532 & 11.78 & \multirow[t]{4}{*}{7.04} & & 0.8822 & 1.185 & 1.675 & \\
\hline & Myopia & 2290 & 657 & 28.69 & & & 0.7131 & 0.553 & 1.180 & 66.57 \\
\hline \multirow[t]{2}{*}{ Girls } & Emmetropia1) & 3930 & 453 & 11.53 & & & 0.8847 & 1.200 & 1.688 & \\
\hline & Myopia & 3028 & 884 & 29.19 & & & 0.7081 & 0.553 & 1.180 & 67.82 \\
\hline \multicolumn{11}{|c|}{ Ethnicity } \\
\hline \multirow[t]{2}{*}{ Han } & Emmetropia ${ }^{1)}$ & 3350 & 479 & 14.30 & \multirow[t]{4}{*}{274.60} & \multirow[t]{4}{*}{$\begin{array}{l}< \\
0.01\end{array}$} & 0.8570 & 1.067 & 1.579 & \\
\hline & Myopia & 3778 & 1205 & 31.90 & & & 0.6810 & 0.468 & 1.118 & 65.02 \\
\hline \multirow[t]{2}{*}{ Other } & Emmetropia ${ }^{1)}$ & 5098 & 506 & 9.93 & & & 0.9007 & 1.287 & 1.760 & \\
\hline & Myopia & 1540 & 336 & 21.82 & & & 0.7818 & 0.772 & 1.346 & 52.71 \\
\hline
\end{tabular}

Multivariate logistic regression models were constructed to evaluate factors associated with myopia after adjusting for confounders. In multivariate models, the presence of myopia was associated with parental myopia, poor reading and writing habits (1), and poor reading and writing habits (2). Both models 1 and 2 were consistent (Table 3).

Table 3

Logistic regression analysis of the correlation of parental myopia and poor reading and writing habits with students' myopia

\begin{tabular}{|lccc|}
\hline & $\boldsymbol{\beta}$ & OR $(95 \%$ CI & $\boldsymbol{P}$ \\
\hline Model 1 & & & $<0.01$ \\
\hline Parental myopia & 1.26 & $3.51(3.11-3.96)$ & $<0.01$ \\
\hline Poor reading and writing habits (1) & 1.03 & $2.81(2.28-3.46)$ & $<0.01$ \\
\hline Poor reading and writing habits (2) & 0.37 & $1.45(1.25-1.68)$ & $<0.01$ \\
\hline Model 2 & & & $<0.01$ \\
\hline Parents myopia & 1.15 & $3.15(2.78-3.57)$ & $<0.01$ \\
\hline Poor reading and writing habits (1) & 1.01 & $2.74(2.21-3.41)$ & \\
\hline Poor reading and writing habits (2) & 0.41 & $1.51(1.29-1.76)$ & \\
\hline Model 1: adjusted for age and sex; Model 2: adjusted for age, sex, ethnicity, and learning level. \\
\hline
\end{tabular}

Parental myopia and poor reading and writing habits (1) and (2) were converted into three dummy variables (DUM). These were then used in the multivariate logistic regression models to obtain three regression coefficients in terms of dummy variables, $O R$ (Table 4), and covariance. Three indicators for evaluating interaction were determined through regression coefficients and covariance: relative excess risk due to interaction $(R E R)$, attributable proportion $(A P)$, and synergy index 
(S) (Table 5). The judgment of additive interaction is based on RERI, AP, S and their $95 \%$ confidence intervals (95\% Cl); when the $95 \% \mathrm{Cl}$ of $R E R /$ and $A P$ does not contain 0 and the $95 \% \mathrm{Cl}$ of $S$ does not contain 1, it indicates that an additive interaction exists and is significant. In models 1 and 2, the $95 \% C l$ of $R E R /$ and $A P$ for parental myopia and both poor reading and writing habits (1) and (2) did not contain 0 and the $95 \% \mathrm{Cl}$ of $S$ did not contain 1, proving that there were additive interactions between them, i.e., when parental myopia and poor reading and writing habits were present at the same time, the risk of having myopia was greater than that due to the existence of the two independently (the additive interaction diagram of Model 2 is shown in Figs. 1 and 2).

Table 4

Analysis of the additive interaction of parental inheritance and poor reading and writing habits on students' myopia

\begin{tabular}{|c|c|c|c|c|c|c|}
\hline & $\beta_{1}$ & $O R_{1}(95 \% C l)$ & $P_{1}$ & $\beta_{2}$ & $O R_{2}(95 \% C l)$ & $P_{2}$ \\
\hline \multicolumn{7}{|c|}{ Parental myopia and poor reading and writing habits (1) } \\
\hline DUM 1 & 1.69 & $5.43(3.60-8.20)$ & $<0.01$ & 1.49 & $4.44(2.90-6.78)$ & $<0.01$ \\
\hline DUM 2 & 1.33 & $3.76(2.91-4.94)$ & $<0.01$ & 1.27 & $3.58(2.74-4.73)$ & $<0.01$ \\
\hline DUM 3 & 2.55 & $12.83(9.81-17.01)$ & $<0.01$ & 2.40 & $10.99(8.33-14.68)$ & $<0.01$ \\
\hline \multicolumn{7}{|c|}{ Parental myopia and poor reading and writing habits (2) } \\
\hline DUM 1 & 1.47 & $4.38(3.38-5.68)$ & $<0.01$ & 1.26 & $3.53(2.70-4.62)$ & $<0.01$ \\
\hline DUM 2 & 0.65 & $1.91(1.60-2.30)$ & $<0.01$ & 0.63 & $1.88(1.56-2.27)$ & $<0.01$ \\
\hline DUM 3 & 1.89 & $6.59(5.43-8.04)$ & $<0.01$ & 1.78 & $5.92(4.84-7.27)$ & $<0.01$ \\
\hline
\end{tabular}

Table 5

Evaluation index for additive interaction between parental inheritance and poor reading and writing habits

\begin{tabular}{|c|c|c|c|}
\hline & $R E R I(95 \% C l)$ & $A P(95 \% C D)$ & $s(95 \% C l)$ \\
\hline \multicolumn{4}{|c|}{ Parental myopia and poor reading and writing habits (1) } \\
\hline Model 1 & $254.23(36.39-427.06)$ & $0.97(0.95-0.98)$ & $36.32(22.86-57.71)$ \\
\hline Model 2 & $167.26(19.37-315.15)$ & $0.96(0.94-0.98)$ & $28.83(18.13-45.83)$ \\
\hline \multicolumn{4}{|c|}{ Parental myopia and poor reading and writing habits (2) } \\
\hline Model 1 & $49.94(20.93-78.95)$ & $0.90(0.87-0.94)$ & $12.63(9.48-16.83)$ \\
\hline Model 2 & $34.87(13.83-55.92)$ & $0.89(0.85-0.92)$ & $11.23(8.49-14.86)$ \\
\hline
\end{tabular}


Table 6

Analysis of multiplicative interaction between parental myopia and poor reading and writing habits on students' myopia

\begin{tabular}{|c|c|c|c|c|c|c|}
\hline & $\beta_{1}$ & $O R_{1}(95 \% C l)$ & $P_{1}$ & $\beta_{2}$ & $O R_{2}(95 \% \mathrm{Cl})$ & $P_{2}$ \\
\hline \multicolumn{7}{|c|}{ Parental myopia and poor reading and writing habits (1) } \\
\hline Parental myopia & 1.69 & $\begin{array}{l}5.43(3.60- \\
8.20)\end{array}$ & 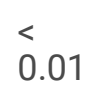 & 1.49 & $\begin{array}{l}4.44(2.90- \\
6.78)\end{array}$ & $<01$ \\
\hline Poor reading and writing habits 1 & 1.33 & $\begin{array}{l}3.76(2.91- \\
4.94)\end{array}$ & 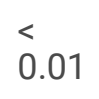 & 1.27 & $\begin{array}{l}3.58(2.74- \\
4.73)\end{array}$ & $<.01$ \\
\hline $\begin{array}{l}\text { Parental myopia and poor reading and writing } \\
\text { habits ( } 1 \text { ) }\end{array}$ & -0.47 & $\begin{array}{l}0.63(0.41- \\
0.96)\end{array}$ & 0.05 & $\overline{0} .37$ & $\begin{array}{l}0.69(0.44- \\
1.08)\end{array}$ & 0.10 \\
\hline \multicolumn{7}{|c|}{ Parental myopia and poor reading and writing habits (2) } \\
\hline Parental myopia & 1.47 & $\begin{array}{l}4.38(3.38- \\
5.68)\end{array}$ & $<01$ & 1.26 & $\begin{array}{l}3.53(2.70- \\
4.62)\end{array}$ & $<.01$ \\
\hline Poor reading and writing habits (2) & 0.69 & $\begin{array}{l}1.91(1.60- \\
2.30)\end{array}$ & 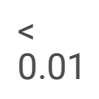 & 0.63 & $\begin{array}{l}1.88(1.56- \\
2.27)\end{array}$ & $<.01$ \\
\hline $\begin{array}{l}\text { Parental myopia and bad reading and writing } \\
\text { habits (2) }\end{array}$ & -0.23 & $\begin{array}{l}0.79(0.59- \\
1.05)\end{array}$ & 0.11 & $\overline{0} .11$ & $\begin{array}{l}0.89(0.66- \\
1.21)\end{array}$ & 0.46 \\
\hline
\end{tabular}

Parental myopia and poor reading and writing habits were entered into the logistic regression model together to obtain the regression coefficient, $O R$, and $P$-value for three variables. After adjusting the relevant variables, if the $P$-value for parental myopia and poor reading and writing habits was less than 0.05 , then it can be considered that parental myopia and poor reading and writing habits have a multiplicative interaction on myopia. However, the $P$ for parental myopia and poor reading and writing habits (1) was less than 0.05 only in Model 1, while in Model 2, the $P$ was greater than 0.05 , and the $P$ for parental myopia and poor reading and writing habits (2) was greater than 0.05 in models 1 and 2; therefore, there were no multiplicative interactions between parental myopia and poor reading and writing habits (2).

\section{Discussion}

This school-based eye survey documented that approximately $19.9 \%$ of primary school students, $44.5 \%$ of junior high school students, and $72.0 \%$ of senior high school had myopia. Although this was lower than the prevalence in most other provinces and cities in China [5], there was a clear upward trend compared with the findings from our research group's survey in 2010 [16, 18]. This upward trend can also be seen in other studies in China [20]. According to Dong et al. [21], the prevalence of myopia in 2050 among children and adolescents aged $3-19$ years is estimated to reach $84 \%$. There is no doubt that if no measures are taken, the rates of myopia in Urumqi primary and middle school students will continue to rise.

Many studies have concluded that girls have a higher prevalence of myopia than boys [21, 22, 23, 24], consistent with our finding. The reason is that girls' learning attitudes are more active than those of boys, and they have less outdoor activities. Sherwin et al. [25] searched 2,912 articles in the MEDLINE, Embase, Web of Science, and CENTRAL databases to conduct a systematic review and meta-analysis and summarized the results of seven cross-sectional studies. The overall results indicated that increasing outdoor activity time might be a strategy to reduce myopia in children and adolescents. Outdoor activities can increase exposure to the sun, and light can stimulate the synthesis and release of dopamine in the retina, which shortens the axis of the eye [26,27], thus preventing myopia; therefore, to reduce the prevalence of myopia, education departments and parents should encourage girls to participate in outdoor activities. Our survey also found that the prevalence of myopia among Han students was higher than that among other ethnic groups. Previous studies by Tang 
et al. [28] reached the same conclusion. The reason for this difference may be owing to differences in lifestyle between the Han and ethnic minority populations in Urumqi. Urumqi is the capital city of Xinjiang, which is a multi-ethnic region. The ethnic minorities living in Xinjiang mainly comprise Uygur, Kazakh, and Hui. These residents have a unique lifestyle. For example, grasp is a staple food unique to Xinjiang. It is made of rice and carrots; carrots are rich in vitamin A, which can prevent corneal dryness and degeneration, eliminate eye fatigue, and protect visual function [29]. In addition, genetic factors may also be responsible for the difference in the prevalence of myopia between sexes and ethnicities; the extent to which genetic factors play a role in disease formation or susceptibility is called heritability. The higher the heritability, the more important the role of genetic factors in the pathogenesis of a disease [16, 30]. Although there was no significant difference between different sexes in our survey, the heritability in the Han population was higher than that in the other ethnic groups, and the heritability for both sexes and ethnicities exceeded $50 \%$, indicating that myopia has a high genetic susceptibility. At present, many studies have shown that having myopic parents increases the risk of myopia in children. For example, a study by O'Donoghue et al. [31] on children aged 12-13 years in Northern Ireland showed that children who have one or both parents with myopia were 2.91 and 7.79 times more likely, respectively, to develop myopia than children whose parents were not myopic. Moreover, some studies have shown that the severity of myopia in parents and the number of myopic parents are factors that lead to aggravation of myopia in children [32, 33]. The multivariate logistic regression in this study also found that parental myopia increases the risk of myopia in children. At present, 25 gene loci are clearly related to the pathogenesis of myopia (MYP1-25) [34,35,36]. With the continuous development of molecular genetics and related detection technologies, an increasing number of genes related to myopia will be found; however, there is still a lack of genetic surveys on myopia in Xinjiang.

In China, students are pressurized to study; therefore, they spend more time reading and writing. Huang et al. [37] searched for articles related to myopia published between 1989 and 2014 in the MEDLINE, Embase, and Cochrane Library databases, found 12 cohort studies and 15 cross-sectional studies, and conducted a meta-analysis that suggested that the time spent reading and writing with the object in close proximity to the eyes was associated with the risk of myopia. If students have poor reading and writing habits, the risk of myopia may be further increased. In this study, multivariate logistic regression found that poor reading and writing habits will increase the risk of myopia. In addition to multivariate logistic regression analysis, we also analyzed the potential additive and multiplicative interaction between parents' heredity and poor reading and writing habits. The definition of interaction is that when two or more risk factors exist at the same time, the risk of the disease is different from the sum of the individual effects of each risk factor [38]. Currently, widely accepted interactions are biological interactions and statistical interactions [39]. Evaluation of statistical interaction is mainly based on additive and multiplicative models. Although statistical interaction does not mean biological interaction, statistical interaction and biological interaction are not completely unrelated. If we have biologically plausible explanation, two factors with a positive additive interaction can indicate a biologically significant synergy, and two factors with negative additive interaction can indicate a biologically significant antagonism [40,41,42,43]. In terms of multiplicative interaction, it is generally believed that if two factors have a positive or no multiplicative interaction, then there will be a positive interaction in the additive model, and if the two factors have a negative multiplicative interaction, there may be three results in the additive model: positive, negative, and no interaction. Therefore, the negative multiplicative interaction cannot indicate whether two factors have a biological interaction. The multiplicative interaction between parental myopia and poor reading and writing habits (1) does not suggest a biological interaction between the two in Model 1. However, this study found that there was an additive interaction between parental myopia and the two kinds of poor reading and writing habits; some scholars have proposed that it is more scientific to use the additive model to analyze biological interaction $[42,44]$. Combined with this study, our conclusions suggest that parental myopia makes children more susceptible to poor reading and writing habits, leading to a greater risk of myopia. It also reminds us that when students enter school, we should investigate whether their parents have myopia, and pay attention to students whose parents have myopia to actively correct poor reading and writing habits to reduce the risk of myopia in students.

\section{Conclusion}


At present, the overall myopia rate of students in Urumqi is relatively high, and myopia has a high genetic susceptibility. For students with parents who have myopia, we should focus on interventions. For students with poor reading and writing habits, schools and parents should remind students to correct these habits to reduce the risk of developing myopia.

\section{Abbreviations}

$C l$ : confidence interval; $O R$ : odds ratio; $R E R$ l: relative excess risk due to interaction; $A P$. attributable proportion; $S$. synergy index

\section{Declarations}

\section{Ethics approval and consent to participate}

This study passed the ethical review of the First Affiliated Hospital of Xinjiang Medical University, and obtained the informed consent of parents and head teachers of all the students investigated.

\section{Consent for publication}

N/A

\section{Availability of data and materials}

The datasets used and/or analysed during the current study available from the corresponding author on reasonable request.

\section{Competing interests}

The authors declare that they have no competing interests.

\section{Funding}

All phases of this study were supported by National Natural Science Foundation of China (grant: 81860179).

\section{Authors'Contributions}

HS analyzed data and wrote the paper, TW designed the study and proofread and reviewed this article.

JF and XC examined the students' eyesight.

$J L$ and $X Y$ was in charge of the investigation

All authors collected the data and were involved in writing the manuscript.

\section{Acknowledgements}

This work was supported by the National Natural Science Foundation of China (grant no. 81860179). We thank the Ophthalmology Department of the First Affiliated Hospital of Xinjiang Medical University for its technical support, and also thank the Urumqi Primary and Secondary School Health Care Guidance Center for its personnel support.

\section{References}

1. Cai X B, Shen S R, Chen D F, et al. An overview of myopia genetics. Experimental eye research, 2019, 107778. 
2. Morgan IG, Ohno-Matsui K, Saw SM. Myopia[J]. Lancet, 2012, 379(9827):1739-1748.

3. Kim H, Seo J S, Yoo W S, et al. Factors associated with myopia in Korean children: Korea National Health and nutrition examination survey 2016-2017 (KNHANES VII)[J]. BMC Ophthalmology, 2020, 20(1).

4. Yotsukura $\mathrm{E}$, Torii $\mathrm{H}$, Inokuchi M, et al. Current prevalence of myopia and association of myopia with environmental factors among schoolchildren in Japan[J]. Jama Ophthalmology, 2019, 137(11): 1233-1239.

5. Zhou J,Ma Y H,Ma J,et al,Prevalence of myopia and influencing factors among primary and middle school students in 6 provinces of China (in Chinese)[J].Chin J Epidemiol,2016,37(1):29-34.

6. Yang G R.Results of the National Survey on Students' Physical Fitness and Health in 2000(in Chinese)[J].Chin J School Health $₫ 2002,23(1): 2-3$.

7. National Student Physical Health Investigation Group.Results of the National Survey on Students' Physical Fitness

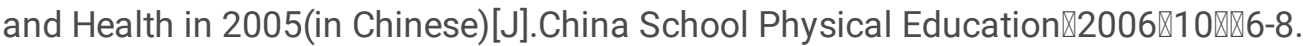

8. Results of the National Survey on Students' Physical Fitness and Health in 2010(in Chinese)[J].Chinese Journal of School Health,2011®32(9):1026.

9. State Physical Culture Administration.Results of the National Survey on Students' Physical Fitness and Health in 2014(in Chinese)[J].Chinese Journal of School Health,2015,IV.

10. Lv F, Chen Q.Research development of myopia in China(in Chinese)[J].Chin J Ophthalmol囚2019(55):153-160.

11. Saw S M,Hong C Y,Chia K S,et al.Nearwork and myopia in young children[J].Lancet,2001,357(9253):390.

12. Chen C J,Cohen B,Diamond E.Genetic and environmental effects on the development of myopia in Chinese twin children[J].Ophthalmol Pediatr Genet,1985,6(1-2):113-119.

13. Goldschmidt E , Jacobsen N. Genetic and environmental effects on myopia development and progression[J]. Eye, 2014, 28(2):126-33.

14. Zhao F,Zhang $D$,Zhou Q,et al.Scleral HIF-1a is a prominent regulatory candidate for genetic and environmental interactions in human myopia pathogenesis[J].EBioMedicine『2020区57:102878.

15. Qian D J,Zhong H,Li J,et al.Myopia among school students in rural China (Yunnan)[J].Ophthalmic and Physiological Optics,2016,36(4)::381-387.

16. Wang T T,Wang Q,Wang Y,et al.Heritability of myopia in Uyhur and Han middle school students in Urumqi city of Xinjiang(in CHinese)[J].Chin J Public Health,2015,31(2):205-207.

17. Wang $T$ T,Munire R,Aikeranmu A,et al.Heritability analysis of myopia among primary and middle school students in Yining City(in Chinese)[J].Chin J Child Health Care,2017,25(8):834-836.

18. Wang $T$ T,Lin $Y$,Wang $X$ T,et al.Prevalence and risk factor of myopia among Uygur and Han junior school students in Urumqi(in Chinese)[J].Chin J Sch Health,2014,35(6):877-880.

19. Lee J H, Jee D , Kwon J W, et al. Prevalence and Risk Factors for Myopia in a Rural Korean Population[J]. Investigative Opthalmology \& Visual Science, 2013, 54(8):5466.

20. Dong $Y \mathrm{H}$,Liu H B,Wang Z H, et al. Prevalence of myopia and increase trend in children and adolescents aged 7-18 years in Han ethnic group in China, 2005-2014(in Chinese)[J]. Chin J Epide,iol,2017,38(5):583-587.

21. Dong L, Kang Y K, Li Y, et al. Prevalence and time trends of myopia in children and adolescents in china: a systemic review and meta-analysis[J]. Retina, 2020, 40(3): 399-411.

22. Rudnicka A R, Kapetanakis V V, Wathern A K, et al. Global variations and time trends in the prevalence of childhood myopia, a systematic review and quantitative meta-analysis: implications for aetiology and early prevention[J]. British Journal of Ophthalmology, 2016, 100(7): 882-890.

23. Schuster A K, Elflein H M, Pokora R, et al. Prevalence and Risk Factors of Myopia in Children and Adolescents in Germany-Results of the KiGGS Survey[J]. Klinische Padiatrie, 2017, 229(4): 234-240. 
24. Wang J, Ying G, Fu X, et al. Prevalence of myopia and vision impairment in school students in Eastern China[J]. BMC ophthalmology, 2020, 20(1): 1-10.

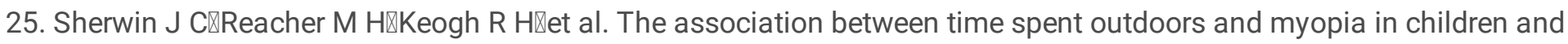
adolescents: a system-atic review and meta-analysis[J]. Ophthalmology,2012,119(10):2141-2151.

26. Rose K A , Morgan I G , Ip J , et al. Outdoor Activity Reduces the Prevalence of Myopia in Children[J]. Ophthalmology, 2008, 115(8):1279-1285.

27. Megaw P L , Boelen M G, Morgan I G , et al. Diurnal patterns of dopamine release in chicken retina[J]. Neurochemistry International, 2006, 48(1):17-23.

28. Tang Y Y, Wang J H, Wang X L. Analysis on the Status and Trend of Myopia Examination Among 7-18 Years old Students of Xinjiang(in Chinese)[J]. J Prec Med Inf, 2019,35(9):1014-1017.

29. Ng F J, Mackey D A, O'Sullivan T A, et al. Is Dietary Vitamin A Associated with Myopia from Adolescence to Young Adulthood?[J]. Translational Vision Science \& Technology, 2020, 9(6): 29-29.

30. Guggenheim J A, Kirov G, Hodson S A. The heritability of high myopia: a reanalysis of Goldschmidt's data[J]. Journal of Medical Genetics, 2000, 37(3): 227-231.

31. O'Donoghue L, Kapetanankis V V, McClelland J F, et al. Risk factors for childhood myopia: findings from the NICER study[J]. Investigative Ophthalmology \& Visual Science, 2015, 56(3): 1524-1530.

32. Xiang F, He M, Morgan I G. The impact of parental myopia on myopia in Chinese children: population-based evidence[J]. Optometry and Vision Science, 2012, 89(10): 1487-1496.

33. Xiang F, He M, Morgan I G. The impact of severity of parental myopia on myopia in Chinese children[J]. Optometry and Vision Science, 2012, 89(6): 884-891.

34. Cai X B, Shen S R, Chen D F, et al. An overview of myopia genetics[J]. Experimental Eye Research, 2019, 188: 107778.

35. Zhao Y Y, Zhang F J, Zhu S Q, et al. The association of a single nucleotide polymorphism in the promoter region of the LAMA1 gene with susceptibility to Chinese high myopia[J]. Molecular vision, 2011,17(112): 1003-1010.

36. Hawthorne F, Feng S, Metlapally R, et al. Association mapping of the high-grade myopia MYP3 locus reveals novel candidates UHRF1BP1L, PTPRR, and PPFIA2[J]. Investigative ophthalmology \& visual science, 2013, 54(3): 20762086.

37. Huang H M, Chang S T, Wu P C. The Association between Near Work Activities and Myopia in Children-A Systematic Review and Meta-Analysis[J]. PLOS ONE, 2015, 10(10):e0140419.

38. MadMahon B, Pugh TE. Epidemiology: principles and methods[M]. Boston Little Brown, 1970.

39. Rothman K J. Epidemiology: An inreoduction[M]. Oxford University Press,2002:168-180.

40. Yuan Y, Li N, REN A G,et al. Analysis of the application of the additive model and the multiplicative statistical model in biological interaction(in Chinese)[J]. Modern Preventive Medicine, 2015,42(6):961-975.

41. Rothman K J, Greenland S. Modern epidemiology, 2nd edition[M]. Lippincott-Raven, Philadelphia, 1998.

42. Rothman K J, Greenland S. Modern epidemiology,3rd edition[M]. Lippincott Williams \& Wilkins, 2008.

43. Ahlbom A, alfredsson L. Interaction: A word with two meanings creates confusion[J]. Eur J Epidemiol, 2005,20(7):563564.

44. Knol M J, Tweel I V D, Grobbee D E, et al. Estimating interaction on an additive scale between continuous determinants in a logistic regression model[J]. International Journal of Epidemiology, 2007, 36(5):1111-1118.

\section{Figures}




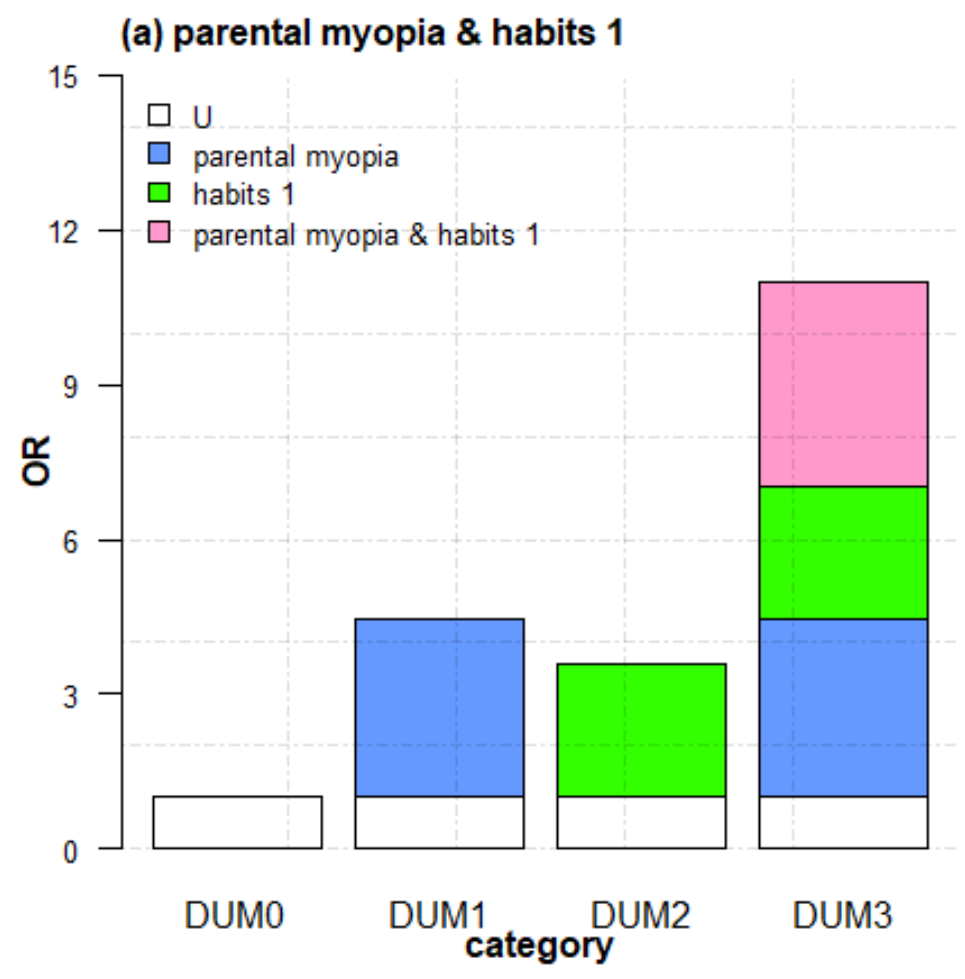

Figure 1

Additive interaction between parental myopia and poor reading and writing habits (1) on myopia

\section{(b) parental myopia \& habits 2}

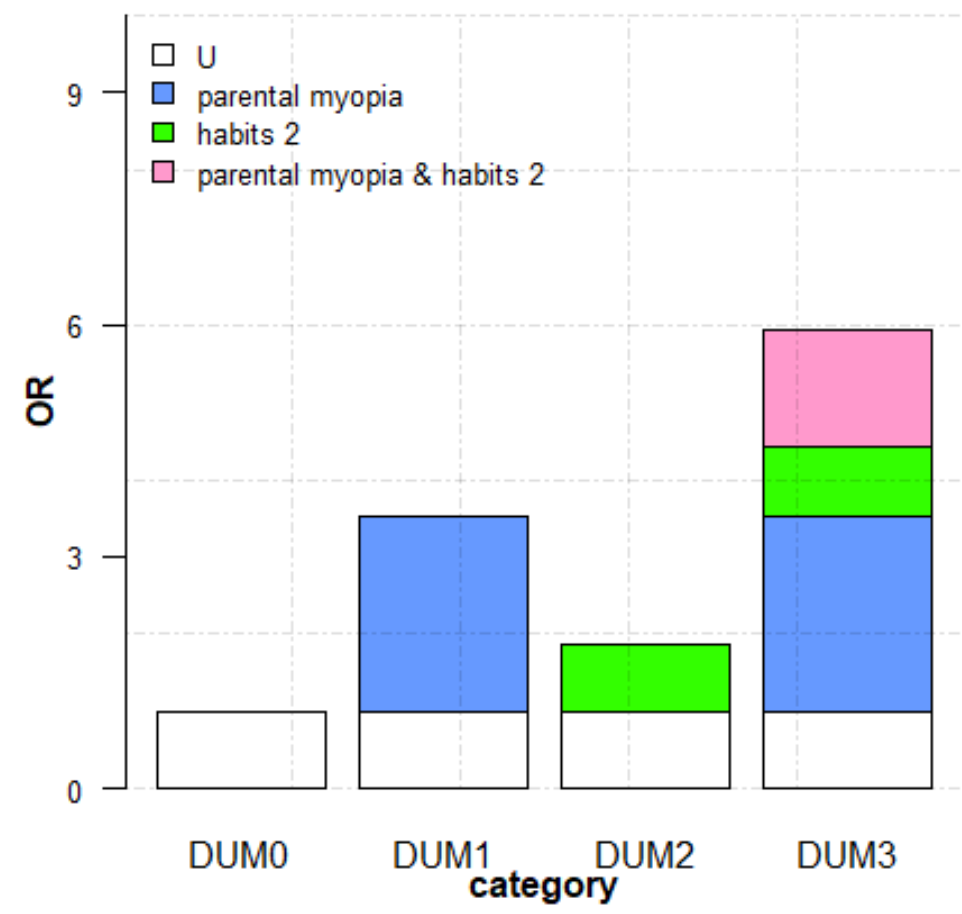

Figure 2

Additive interaction between parental myopia and poor reading and writing habits (2) on myopia 


\section{Supplementary Files}

This is a list of supplementary files associated with this preprint. Click to download.

- Authorshipform.pdf 